\title{
Nasal High-Flow Oxygen and Total Intravenous Anaesthesia for Tubeless Airway Assessment and Surgery in Spontaneously Breathing Children
}

\author{
RG Craig1* , AJ Howie ${ }^{1}$, S Acheson ${ }^{1}$ and R Wilde ${ }^{2}$ \\ ${ }^{1}$ Department of Anaesthesia, Alder Hey Children's Hospital, United Kingdom \\ ${ }^{2}$ Department of Anaesthesia, John Radcliffe Hospital, United Kingdom
}

Submission: August 21, 2019; Published: August 28, 2019

*Corresponding author: Dr RG Craig, Jackson Rees Department of Anaesthesia, Alder Hey Children’s Hospital, Eaton Road, Liverpool, L12 2AP, United Kingdom

*Presented in part at the Association of Paediatric Anaesthetists of Great Britain and Ireland Annual Scientific Meeting in Liverpool on the 18th May 2018

\section{Summary}

Nasal high-flow oxygen can be used in conjunction with propofol and remifentanil total intravenous anaesthesia to facilitate airway endoscopy and surgery in spontaneously breathing children. Nasal high-flow oxygen has been used for Trans nasal Humidified Rapid-Insufflation Ventilatory Exchange in children to prolong the safe apnoea time. As such, it may facilitate the maintenance of oxygenation during hypoventilation and brief periods of apnoea in children undergoing airway endoscopy and airway surgery. This in turn may enable the anaesthetist to increase the depth of anaesthesia during particularly stimulating parts of the procedure as well as reduce the incidence of hypoxaemia and the need to interrupt the procedure for rescue oxygenation. We report our institutional experience following the introduction of nasal high-flow oxygen for this purpose. Data were prospectively collected in 28 children undergoing airway endoscopy and surgery. Following induction, anaesthesia was maintained with propofol and remifentanil infusions titrated to allow laryngoscopy and surgery whilst maintaining spontaneous respiration. Nasal high-flow oxygen was administered using the OptiflowTM nasal cannulae. Topical lidocaine was applied to the larynx and trachea. ECG, non-invasive blood pressure, pulse oximetry, transcutaneous carbon dioxide level (tcCO2) and drug infusion rates were recorded. Adverse events including desaturation requiring interruption of the procedure for rescue oxygenation; coughing; laryngospasm; and apnoea were recorded. The age range was 6 months to 11 years and weight range 5 to $65 \mathrm{~kg}$. The mean lowest recorded saturation was $94.5 \%$ (SD 8.6\%). Rescue oxygenation with interruption of the procedure was required in two cases. Desaturation below $90 \%$ occurred 8 times in 7 different patients (25\%). The mean maximum tcCO2 level was $8.2 \mathrm{KPa}(\mathrm{SD} 1.5 \mathrm{KPa})$. The correlation coefficient for maximum tcCO2 and duration of procedure is $0.226(\mathrm{P}=0.288)$. Episodes of hypoxaemia requiring interruption of the procedure for rescue oxygenation remain relatively common despite the use of nasal highflow oxygen with propofol and remifentanil TIVA. Apnoea is well tolerated without desaturation in patients without severe anatomical airway obstruction. The technique is associated with moderate hypercapnia that is unrelated to the duration of the procedure.

Keywords: Anesthesia; Intravenous; Airway management; Oxygen; Apnoea; Insufflation; Child

\section{Introduction}

The use of nasal high-flow oxygen for the maintenance of oxygenation during anaesthesia for tubeless airway procedures in spontaneously breathing children with abnormal airways has recently been reported [1]. We conducted a service evaluation project to introduce this technique in our institution. We report on our experience using nasal high-flow oxygen and total intravenous anaesthesia (TIVA) in 28 spontaneously breathing children undergoing microlaryngotracheobronchoscopy (MLTB) and upper airway surgery.

For dynamic airway assessment using suspension laryngoscopy and a Hopkins telescope, the surgeon requires an unobstructed view of the larynx and trachea in a child who is breathing spontaneously. Depth of anaesthesia must be titrated such that the child tolerates laryngoscopy, airway instrumentation, and surgery whilst avoiding apnoea. Various anaesthetic techniques can achieve this. In our institution two techniques are commonly used: inhalational anaesthesia with sevoflurane in oxygen delivered via a nasopharyngeal airway; and total intravenous anaesthesia using propofol and remifentanil with oxygen delivered via a nasopharyngeal airway. With both techniques, topical lidocaine is sprayed onto the larynx and down the trachea to facilitate airway endoscopy and surgery. Choice of technique is dependent on the personal preference of the anaesthetist. 
The use of nasal high-flow oxygen instead of a nasopharyngeal airway in patients receiving total intravenous anaesthesia was an incremental change in practice which we introduced by way of a formal service evaluation. Nasal high-flow oxygen cannot be used in conjunction with sevoflurane anaesthesia because the high flow rates will flush the sevoflurane from the pharynx, and it will not be possible to maintain anaesthesia.

Placing a nasopharyngeal airway can cause epistaxis, particularly in children with large adenoids. The use of nasal high-flow oxygen in conjunction with TIVA avoids this problem. It may also reduce the likelihood of desaturation requiring interruption of the procedure for rescue oxygenation [1]. Nasal high flow oxygen has been used for Trans nasal Humidified RapidInsufflation Ventilatory Exchange in children to prolong the safe apnoea time [2]. As such, it may facilitate the maintenance of oxygenation during hypoventilation and brief periods of apnoea in children undergoing airway endoscopy and airway surgery [1]. This in turn may enable the anaesthetist to increase the depth of anaesthesia during particularly stimulating parts of the procedure, although careful monitoring of carbon dioxide clearance will be required. End-tidal carbon dioxide cannot be measured during TIVA with nasal high-flow oxygen. Transcutaneous carbon dioxide measurement allows monitoring of carbon dioxide levels in this situation. Transcutaneous carbon dioxide has been shown to correlate well with the partial pressure of arterial carbon dioxide (PaCO2) with no detectable difference between the two measurements [3].

\section{Methods}

The project was reviewed by the institutional Quality and Governance Department and classified as a service evaluation. Data were collected as part of delivering standard care following the introduction of a new technique into clinical practice. Between January and July 2018, we collected data on children presenting for microlaryngotracheobronchoscopy and upper airway surgery for who the anaesthetist used total intravenous anaesthesia and nasal high-flow oxygen. We excluded children having laser surgery, children with a tracheostomy and children weighing less than $5 \mathrm{~kg}$.

\section{Anaesthetic technique}

Anaesthesia was induced with either sevoflurane in oxygen or intravenous propofol. Monitoring was established immediately upon induction with SpO2, ECG, non-invasive blood pressure, and tcCO2. Transcutaneous carbon dioxide was monitored using the TCM5 monitor (Radiometer, Crawley, England) with the sensor attached to the earlobe.

Maintenance of anaesthesia was with propofol and remifentanil infusions. Propofol was administered as a target-controlled infusion (TCI) using the Paedfusor model and remifentanil was administered as an infusion in micrograms.kg-1.min-1. Both were titrated to maintain spontaneous ventilation and allow laryngoscopy and surgery.
After induction of anaesthesia and commencing TIVA, nasal high-flow oxygen was applied using OptiflowTM nasal cannulae (Fisher \& Paykel Healthcare, Auckland, New Zealand). The RT330 breathing circuit, an MR850 humidifier, and 0-30l.min-1 flowmeter were used in conjunction with the paediatric OptiflowTM Junior cannulae. The AA400 circuit, an MR810 humidifier, and a 0-70l. min-1 flowmeter were used in conjunction with the small, medium or large adult AA001 filtered nasal cannulae. Nasal cannulae were sized according to the manufacturer's recommendations so that there is a clear space around the prong in the nares and the prongs occlude $50 \%$ of the area of the nares. Opti flow junior nasal cannulae come in four different sizes: premature (maximum flow rate 81.min-1); neonatal (maximum flow rate 8l.min-1); infant (maximum flow rate 20l.min-1); and paediatric (maximum flow rate 25l.min-1). When using the Opti flow Junior Nasal Cannulae the flow rate was set at the prescribed maximum for the size of cannula. When using the adult AA001 filtered nasal cannulae, the flow rate was set at 50l.min-1. 100\% oxygen was administered in all cases.

Prior to direct laryngoscopy we checked response to jaw thrust - any response to this manoeuvre resulted in an increase in propofol and/or remifentanil infusion rates and more time was allowed to achieve an appropriate depth of anaesthesia. Direct laryngoscopy was performed and topical 2\% lidocaine $4 \mathrm{mg}$. kg-1 was applied to the laryngeal inlet using a MADgic LaryngoTracheal Mucosal Atomization Device (Teleflex, Athlone, Ireland). Jaw support was maintained until the surgeon inserted a tubular laryngoscope for suspension.

\section{Data collection}

Age, weight, American Society of Anaesthesiologists physical status classification, reason for surgery, procedure performed, method of induction, range of maintenance infusion rates for propofol and remifentanil, lowest oxygen saturation, episodes of desaturation requiring interruption of the procedure for rescue oxygenation, maximum tcCO2, and duration of the procedure were recorded. Rescue oxygenation was defined as a rapid decline in Sp02 below 90\% which required interruption of the procedure for bag-mask ventilation or intubation [1]. All incidences of coughing, laryngospasm, apnoea lasting more than 20 seconds, and desaturation below $90 \%$ as well as any interventions in response to these events were documented.

\section{Results}

We collected data on 28 children undergoing microlaryngotracheobronchoscopy and airway surgery. The median age was 3 years (range 6 months to 11 years). The median weight was $14 \mathrm{~kg}$ (range $5-65 \mathrm{~kg}$ ). Six children were ASA 1, eighteen were ASA 2, and four were ASA 3. Seventeen children had a Cormack and Lehane grade 1 view on laryngoscopy, nine patients had a grade 2 view, and two patients had a grade 3 view. The airway pathology and procedure performed for each patient is shown in Table 1, along with the lowest recorded SpO2 and maximum tcCO2. 


\section{Journal of Anesthesia \& Intensive Care Medicine}

The mean lowest saturation in all patients was $94.5 \%$ (SD 8.6\%). Rescue oxygenation with interruption of the surgical procedure was required in two cases: a child with laryngeal papillomata causing complete obstruction of the glottis who became apnoeic and desaturated to $77 \%$ requiring intubation; and an infant with a history of preterm birth that developed laryngospasm during resection of a subglottic cyst requiring bagmask ventilation with CPAP. Desaturation below $90 \%$ occurred 8 times in 7 different patients (25\%; Table 1).

The maximum tcCO2 had a mean of $8.2 \mathrm{kPa}$ (SD $1.5 \mathrm{kPa}$ ). The correlation coefficient for maximum tcCO2 and duration of procedure is $0.226(\mathrm{P}=0.288)$ (Figure 1$)$.

Table 1: Patient characteristics; MLTB=Microlaryngotracheobronchoscopy; PICU=Paediatric Intensive Care Unit; OSA=Obstructive Sleep Apnoea; $\mathrm{CP}=$ Cerebral Palsy; GDD=Global Developmental Delay

\begin{tabular}{|c|c|c|c|c|c|c|}
\hline Pat \# & Age & Reason for Surgery & Procedure Performed & $\begin{array}{c}\text { Duration } \\
\text { (min) }\end{array}$ & $\begin{array}{l}\text { Max tcCO2 } \\
\text { (KPa) }\end{array}$ & $\begin{array}{c}\text { Min } \\
\text { Sp02(\%) }\end{array}$ \\
\hline 1 & 4 years & $\begin{array}{l}\text { Follow-up after laryngotracheal } \\
\text { reconstruction for subglottic stenosis }\end{array}$ & MLTB & 21 & 6.8 & 98 \\
\hline 2 & 6 months & $\begin{array}{l}\text { Laryngomalacia and retroverted } \\
\text { epiglottis }\end{array}$ & MLTB+supraglottoplasty & 19 & 7.9 & 100 \\
\hline 3 & 3 years & $\begin{array}{l}\text { Recurrent croup and recurrent } \\
\text { tonsillitis }\end{array}$ & MLTB+adenotonsillectomy & 26 & 7.9 & 97 \\
\hline 4 & 10 months & Subglottic haemangioma & MLTB & 26 & 7.1 & 82 \\
\hline 5 & 9 months & Noisy breathing, adenoid hypertrophy & MLTB+adenoidectomy & 26 & 9.8 & 96 \\
\hline 6 & 2 years & $\begin{array}{l}\text { Pedunculated lesion at the base of the } \\
\text { tongue - salivary gland choristoma }\end{array}$ & MLTB+excision biopsy & 26 & 6.1 & 100 \\
\hline 7 & 3 years & Moebius syndrome, subglottic stenosis & MLTB & 31 & 10.5 & 66 \\
\hline 8 & 10 years & Recurrent croup & MLTB & 26 & 8.9 & 100 \\
\hline 9 & 1 year & $\begin{array}{l}\text { Recurrent aspiration and episodes of } \\
\text { cyanosis. To rule out a laryngeal cleft. }\end{array}$ & MLTB (no laryngeal cleft) & 32 & 6.0 & 100 \\
\hline 10 & 1 year & Laryngomalacia & $\begin{array}{l}\text { MLTB+supraglottoplasty } \\
\text { and aryepiglottoplasty }\end{array}$ & 54 & 7.0 & 98 \\
\hline 11 & 6 months & Tracheal stenosis & MLTB+balloon dilatation & 34 & 8.0 & 84 \\
\hline 12 & 1 year & Laryngomalacia & MLTB+aryepiglottoplasty & 34 & 8.1 & 99 \\
\hline 13 & 1 year & Foreign body aspiration & $\begin{array}{l}\text { MLTB+retrieval of foreign } \\
\text { body (peanut) }\end{array}$ & 72 & Unavailable & 97 \\
\hline 14 & 11 years & $\begin{array}{l}\text { Recurrent respiratory papillomatosis } \\
\text { and tracheomalacia }\end{array}$ & MLTB+microdebridement & 55 & Unavailable & 99 \\
\hline 15 & 7 years & Recurrent respiratory papillomatosis & MLTB+microdebridement & 50 & 10.8 & 77 \\
\hline 16 & 3 years & $\begin{array}{l}\text { Aspiration on videofluoroscopy. } \\
\text { MLTB to rule out laryngeal cleft. } \\
\text { Demonstrated left vocal cord palsy. }\end{array}$ & MLTB & 34 & 8.0 & 99 \\
\hline 17 & 11 months & $\begin{array}{l}\text { Ex-prem; multiple PICU admissions; } \\
\text { subglottic cysts }\end{array}$ & $\begin{array}{l}\text { MLTB+excision of } \\
\text { subglottic cysts }\end{array}$ & 48 & 9.0 & 84 \\
\hline 18 & 7 months & Subglottic haemangioma & MLTB & 28 & 8.4 & 86 \\
\hline 19 & 6 years & $\begin{array}{l}\text { Recurrent croup requiring PICU } \\
\text { admission }\end{array}$ & MLTB & 31 & 7.3 & 100 \\
\hline 20 & 3 years & Recurrent croup & MLTB & 27 & 8.4 & 98 \\
\hline 21 & 1 year & To investigate possible laryngomalacia & $\begin{array}{c}\text { MLTB+EUA } \\
\text { PNS+grommets }\end{array}$ & 29 & 7.1 & 99 \\
\hline 22 & 3 years & Ex-prem; stertor, OSA & & & & \\
\hline $\begin{array}{c}3 \text { small } \\
\text { subglottic } \\
\text { cysts; } \\
\text { moderate } \\
\text { adenoid } \\
\text { hypertrophy; } \\
\text { grade } 2 \\
\text { tonsils }\end{array}$ & $\begin{array}{c}\text { MLTB+ } \\
\text { adenotonsillectomy }\end{array}$ & 25 & 9.0 & 100 & & \\
\hline 23 & 11 years & $\begin{array}{l}\text { Noisy breathing and upper airway } \\
\text { obstruction; CP; epilepsy; GDD }\end{array}$ & MLTB & 19 & 9.8 & 98 \\
\hline
\end{tabular}


Journal of Anesthesia \& Intensive Care Medicine

\begin{tabular}{|c|c|c|c|c|c|c|}
\hline 24 & 3 years & Recurrent croup & MLTB & 21 & 9.3 & 100 \\
\hline 25 & 10 years & Recurrent respiratory papillomatosis & MLTB+microdebridement & 55 & 12.1 & 89 \\
\hline 26 & 4 years & Recurrent croup & MLTB & 25 & 7.0 & 100 \\
\hline 27 & 1 year & Subglottic stenosis & MLTB & 31 & 6.4 & 99 \\
\hline 28 & 4 years & Recurrent croup & MLTB & 29 & 7.2 & 100 \\
\hline Mean(SD) & & & & $33(13)$ & $8.2(1.5)$ & $94.5(8.6)$ \\
\hline
\end{tabular}

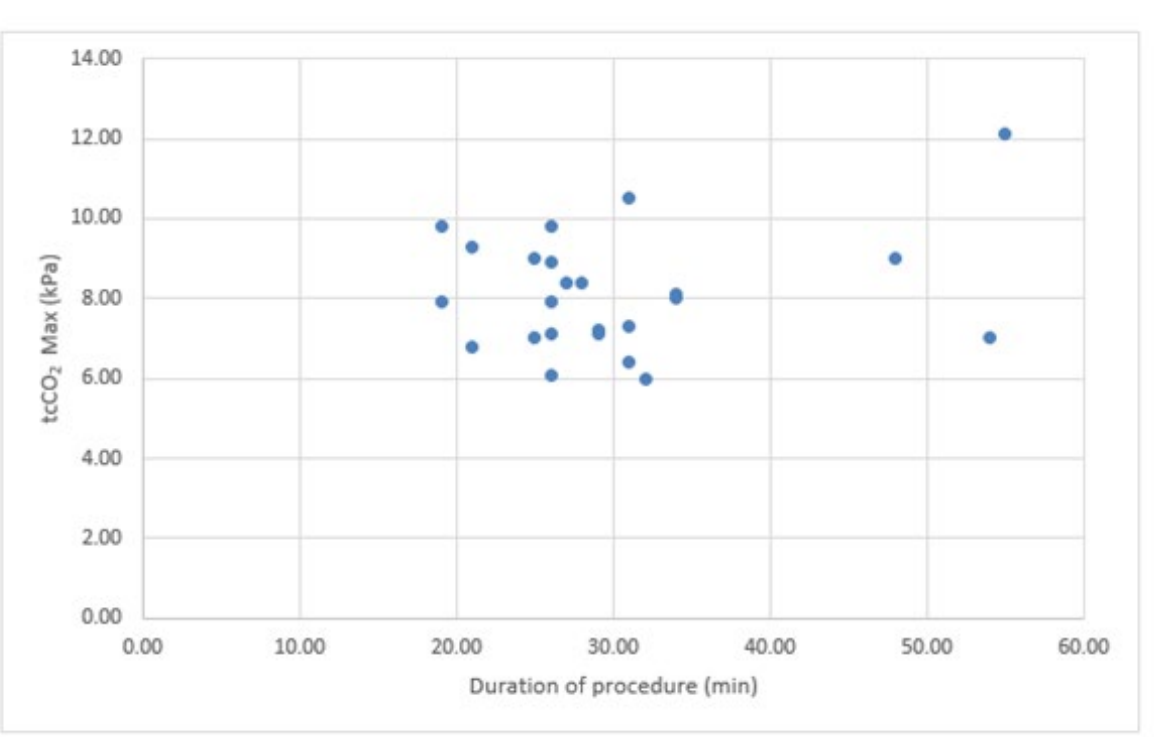

Figure1: The relationship between the duration of the procedure, measured in minutes from the start of induction to the end of the surgical procedure, and the transcutaneous carbon dioxide level (tcCO2) measured in $\mathrm{KPa}$.

Five episodes of apnoea lasting greater than 20 seconds occurred in four different patients (14\%). In 2 episodes this was associated with desaturation; both in patients with laryngeal papillomatosis causing significant airway obstruction. Eleven episodes of coughing occurred in eight patients (29\%). Four episodes coughing occurred on spraying the cords with local anaesthetic. One episode required brief withdrawal of the telescope before continuing. One patient had laryngospasm as described above. No patients had epistaxis.

The median duration of the procedure, from induction of anaesthesia to the end of surgery, was $29 \mathrm{~min}$ (range 19-72min). The median interval from the start of induction until topical lidocaine was applied to the laryngeal inlet and trachea was 13min (range 6-25min). Twenty-four children had an inhalational induction with sevoflurane in oxygen before conversion to TIVA. During the maintenance phase of the procedure, after topicalization of the airway, the lowest propofol target plasma concentration had a median value of $3.5 \mu \mathrm{g}$.ml-1 (range $2-7 \mu \mathrm{g}$. ml-1). The highest propofol target plasma concentration had a median value of $4.5 \mu \mathrm{g} . \mathrm{ml}-1$ (range $2.5-8 \mu \mathrm{g} . \mathrm{ml}-1$ ). The lowest remifentanil infusion rate had a median value of $0.06 \mu \mathrm{g} . \mathrm{kg}-1$.min1 , with a range of $0.02-0.1 \mu \mathrm{g} . \mathrm{kg}-1 . \mathrm{min}-1$. The highest remifentanil infusion rate had a median value of $0.08 \mu \mathrm{g} . \mathrm{kg}-1 . \mathrm{min}-1$, with a

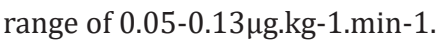

\section{Discussion}

Nasal high-flow oxygen can be used to deliver oxygen during upper airway endoscopy and surgery with TIVA titrated to maintain anaesthesia and spontaneous ventilation. An observational study describing the use of nasal high-flow oxygen during anaesthesia in twenty spontaneously breathing children reported one episode of rescue oxygenation requiring interruption of the procedure and a mean lowest oxygen saturation of $96 \%$ [1]. Twelve of the twenty patients had tubeless airway surgery, four patients underwent flexible bronchoscopy, the remainder had either an expected difficult airway or co-morbidity related risk of apnoea. The episode requiring rescue oxygenation was in a patient having tubeless airway surgery. We encountered two episodes where the procedure was interrupted to provide rescue oxygenation in 28 patients, all having tubeless airway procedures. The lowest recorded oxygen saturation in our series had a mean value of $94.5 \%$.

Malherbe et al. [4] reported their findings in 41 children who underwent 52 airway endoscopies and airway surgeries using propofoland remifentanil total intravenous anaesthesia. Thirty-five of these procedures were microlaryngotracheobronchoscopies. The remaining seventeen were rigid bronchoscopies. Oxygen was administered via the side arm of the suspension laryngoscope or bronchoscope. They reported a mean lowest oxygen saturation of 
93.4\%. Three patients required assisted ventilation for apnoea and two patients required assisted ventilation for desaturation below $90 \%$. Desaturation below $90 \%$ was encountered in 10 children (19\%) [4]. We observed desaturation below $90 \%$ in 7 out of 28 cases (25\%). Shen et al. [5] described using TIVA with propofol and remifentanil for foreign body removal with oxygenation via the side arm of the bronchoscope: desaturation below $90 \%$ occurred in 10 out of 60 cases (16.7\%). Only one patient in our cohort had a rigid bronchoscopy as part of the airway endoscopy. In this case oxygen was administered via the side arm after insertion of the bronchoscope; nasal high flow oxygen was administered during laryngoscopy before and after bronchoscopy.

Apnoea was well tolerated in the absence of significant anatomical airway obstruction. One patient was apnoeic for the entire procedure (15 minutes) without a drop-in oxygen saturation below $98 \%$. Apnoea can be safely prolonged through the use of high-flow oxygen [2]. However, one patient with severe glottic obstruction due to laryngeal papillomatosis became apnoeic and desaturated despite nasal high-flow oxygen, requiring intubation to rescue oxygenation. Four patients had a significant apnoea during the procedure (14\%) compared to $21 \%$ in the study by Malherbe et al. [4]. The mean maximum remifentanil rate in the latter was 0.14 micrograms.kg-1.min-1, which is higher than the rate of infusion in our series.

In spontaneously breathing children the maximum tcCO2 does not correlate with the duration of the procedure. The highest tcCO2 recorded in our series was $12.1 \mathrm{kPa}$. The mean highest $\mathrm{CO} 2$ was $8.2 \pm 1.5 \mathrm{kPa}$. Malherbe et al. [4] reported a mean highest tcCO2 of $8.37 \pm 2.04 \mathrm{kPa}$. The use of high flow oxygen does not appear to reduce the maximum carbon dioxide level compared to oxygen insufflation via the side arm of the bronchoscope or the suspension laryngoscope. A study involving 323 adult patients undergoing general anaesthesia for bronchoscopic interventions showed that moderate hypercapnia $(\mathrm{PaCO} 2<13.3 \mathrm{KPa})$ was not associated with delayed recovery or any other complications [6]. We did not observe any problems related to hypercapnia.

This series represents a formal evaluation of the introduction of NHF oxygen for use with TIVA in children having airway endoscopy in our institution. Our experience has prompted several refinements of the technique which are the subject of an ongoing audit process. In this series we selected the size of the nasal cannula based on the size of the child's nares in accordance with the manufacturer's advice. We also respected the maximum flow rate specified by the manufacturer for the various sizes of Opti flow junior cannulae. Furthermore, the Opti flow junior nasal cannulae are used in conjunction with a circuit and flow meter that is limited to 30l.min-1. A weight-related flow rate has been used in other studies of NHF oxygen and THRIVE in children [1,2]. This requires that the small adult nasal cannula be used for all children $>15 \mathrm{~kg}$. We now adhere to the flow rates published by Humphreys et al. [1]. It takes time to achieve an adequate depth of anaesthesia for airway instrumentation. We now allow 15 minutes from the start of anaesthesia before laryngoscopy and topical lidocaine application. In addition to this we monitor Bispectral index (BiS), targeting a BIS of 40-60, and respiratory rate, aiming for a $50 \%$ reduction in respiratory rate or a rate of 10 breaths per minute in older children.

Several anaesthetic techniques for tubeless airway surgery in the spontaneously breathing child have been described: techniques using sevoflurane in oxygen; propofol and remifentanil TIVA; and hybrid techniques using sevoflurane and propofol [7-9]. The use of propofol and remifentanil TIVA with nasal high flow oxygen does not appear to reduce the likelihood of desaturation. It does avoid epistaxis which is often associated with the placement of a nasopharyngeal airway. A randomised control trial comparing nasal high flow oxygen with propofol and remifentanil TIVA to alternative techniques is required to evaluate differences in the incidence of desaturation, interruption of the procedure for rescue oxygenation, coughing, laryngospasm, apnoea and other adverse events. A clinically significant difference is required to justify the additional cost of equipment and disposables for the delivery of nasal high flow oxygen.

In summary, episodes of hypoxaemia requiring interruption of the procedure for rescue oxygenation remain relatively common despite the use of nasal high flow oxygen with propofol and remifentanil TIVA. Apnoea is well tolerated without desaturation in patients without severe anatomical airway obstruction. A randomised control trial is required to compare this technique to alternatives to look for clinically significant differences.

\section{Disclosures}

\section{Ethical approval}

The project was reviewed by the institutional Quality and Governance Department and classified as a service evaluation. Data were collected as part of delivering standard care following the introduction of a new technique into clinical practice; an incremental change in practice. I have attached the approval letter from the institutional Head of Quality, Quality and Governance Manager.

\section{References}

1. Humphreys S, Rosen D, Housden T, Taylor J, Schibler A (2017) Nasal High-Flow Oxygen Delivery in Children with Abnormal Airways. Paediatr Anaesth 27(6): 616-620.

2. Humphreys S, Lee-Archer P, Reyne G, Long D, Williams T, et al. (2017) Transnasal Humidified Rapid-Insufflation Ventilatory Exchange (THRIVE) in Children: A Randomized Controlled Trial. Br J Anaesth 118(2): 232-238.

3. Gustafsson IM, Lodenius A, Tunelli J, Ullman J, Jonsson FM (2017) Apnoeic Oxygenation in Adults Under General Anaesthesia Using Transnasal Humidified Rapid-Insufflation Ventilatory Exchange (THRIVE) - A Physiological Study. Br J Anaesth 118(4): 610-617.

4. Malherbe S, Whyte S, Singh P, Amari E, King A, et al. (2010) Total Intravenous Anaesthesia and Spontaneous Respiration for Airway Endoscopy in Children- A Prospective Evaluation. Paediatr Anaesth 20(5): 434-438. 
5. Shen X, Hu CB, Ye M, Chen YZ (2012) Propofol-Remifentanil Intravenous Anesthesia and Spontaneous Ventilation for Airway Foreign Body Removal in Children with Pre-Operative Respiratory Impairment. Paediatr Anaesth 22(12): 1166-1170.

6. Cheng Q, Zhang J, Wang H, Zhang R, Yue Y, et al. (2015) Effect of Acute Hypercapnia on Outcomes and Predictive Risk Factors for Complications among Patients Receiving Bronchoscopic Interventions under General Anaesthesia. PLoS One 10: e0130771.

7. Chai J, Wu XY, Han N, Wang LY, Chen WM (2014) A Retrospective Study of Anesthesia During Rigid Bronchoscopy for Airway Foreign Body
Removal in Children: Propofol and Sevoflurane with Spontaneous Ventilation. Paediatr Anaesth 24(10): 1031-1036.

8. Liao R, Li JY, Liu GY (2010) Comparison of Sevoflurane Volatile Induction/Maintenance Anaesthesia and Propofol-Remifentanil Total Intravenous Anaesthesia for Rigid Bronchoscopy under Spontaneous Breathing for Tracheal/Bronchial Foreign Body Removal in Children. Eur J Anaesthesiol 27(11): 930-934.

9. Fidkowski CW, Zheng H, Firth PG (2010) The Anesthetic Considerations of Tracheobronchial Foreign Bodies in children: A Literature Review of 12,979 Cases. Anesth Analg 111(4): 1016-1025.

Your next submission with Juniper Publishers will reach you the below assets

- Quality Editorial service

- Swift Peer Review

- Reprints availability

- E-prints Service

- Manuscript Podcast for convenient understanding

- Global attainment for your research

- Manuscript accessibility in different formats

( Pdf, E-pub, Full Text, Audio)

- Unceasing customer service

Track the below URL for one-step submission https://juniperpublishers.com/online-submission.php 